\title{
Nutritionally Variant Streptococcus
}

National Cancer Institute

\section{Source}

National Cancer Institute. Nutritionally Variant Streptococcus. NCI Thesaurus. Code C123554.

A bacterium that is assigned to the genus Streptococcus that is incapable of supporting its own growth on standard culture plates but can sustain growth using substances released into the medium by the dominant colonies. These Streptococci require Lcysteine for growth. They have been identified as an etiologic agent of endocarditis and other infections. 